\title{
Inhaltsverzeichnis
}

Vorwort $\ldots \ldots \ldots \ldots \ldots \ldots \ldots \ldots \ldots \ldots \ldots \ldots \ldots \ldots$

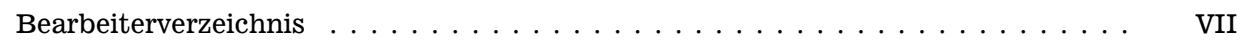

Verzeichnis der abgekürzt zitierten Literatur $\ldots \ldots \ldots \ldots \ldots \ldots$ XVII

Abkürzungsverzeichnis $\ldots \ldots \ldots \ldots \ldots \ldots \ldots \ldots \ldots \ldots \ldots$

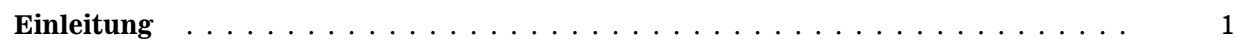

\section{Einkommensteuergesetz (EStG)}

\section{Steuerpflicht}

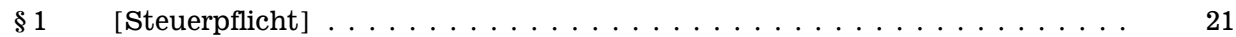

§ 1a [Fiktive unbeschränkte Steuerpflicht von EU-/EWR-Angehörigen] . . . . . . 32

\section{Einkommen}

1. Sachliche Voraussetzungen für die Besteuerung

$\S 2$ Umfang der Besteuerung, Begriffsbestimmungen . . . . . . . . . . . . 37

$\S 2 \mathrm{a}$ Negative Einkünfte mit Bezug zu Drittstaaten . . . . . . . . . . . . $\quad 71$

\section{Steuerfreie Einnahmen}

$\S 3 \quad$ Steuerfreie Einahmen $\ldots \ldots \ldots \ldots \ldots \ldots \ldots \ldots \ldots \ldots$

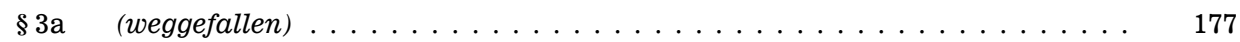

$\S 3 \mathrm{~b} \quad$ Steuerfreiheit von Zuschlägen für Sonntags-, Feiertags- oder Nachtarbeit . . 177

$\S 3 \mathrm{c}$ Anteilige Abzüge $\ldots \ldots \ldots \ldots \ldots \ldots \ldots \ldots \ldots \ldots$

\section{Gewinn}

$\S 4 \quad$ Gewinnbegriff im Allgemeinen . . . . . . . . . . . . . . . . . 201





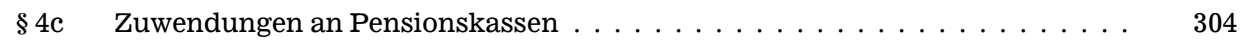

$\S 4 \mathrm{~d}$ Zuwendungen an Unterstützungskassen $\ldots \ldots \ldots \ldots \ldots \ldots \ldots \ldots$

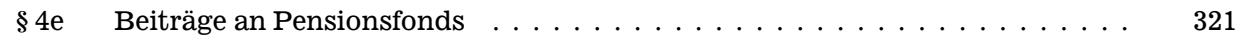

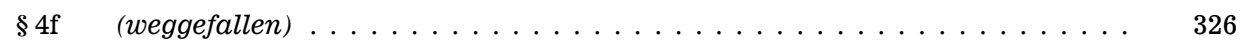

$\S 4 \mathrm{~g} \quad$ Bildung eines Ausgleichspostens bei Entnahme nach $\S 4$ Absatz 1 Satz $3 \ldots \ldots 26$

$\S 4 \mathrm{~h} \quad$ Betriebsausgabenabzug für Zinsaufwendungen (Zinsschranke) . . . . . . . 333

$\S 5 \quad$ Gewinn bei Kaufleuten und bei bestimmten anderen Gewerbetreibenden . . 350

§ 5a Gewinnermittlung bei Handelsschiffen im internationalen Verkehr . . . . . . 413

$\S 5 \mathrm{~b} \quad$ Elektronische Übermittlung von Bilanzen sowie Gewinn- und Verlustrech-

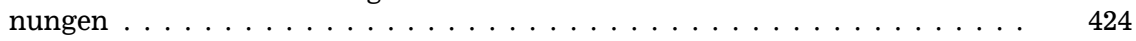

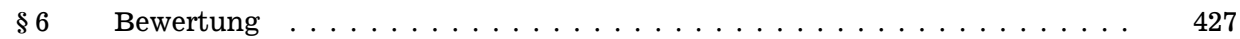

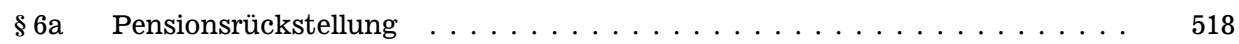

§ 6b Übertragung stiller Reserven bei der Veräußerung bestimmter Anlagegüter . $\quad 538$

§ 6c Übertragung stiller Reserven bei der Veräußerung bestimmter Anlagegüter bei der Ermittlung des Gewinns nach $\S 4$ Absatz 3 oder nach Durchschnittssätzen .......................... 566

$\S 6 \mathrm{~d} \quad$ Euroumrechnungsrücklage $\ldots \ldots \ldots \ldots \ldots \ldots \ldots \ldots$

$\S 7 \quad$ Absetzung für Abnutzung oder Substanzverringerung $\ldots \ldots \ldots \ldots$ 
§ 7a Gemeinsame Vorschriften für erhöhte Absetzungen und Sonderabschreibun-

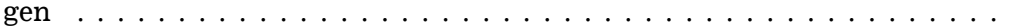 \\ $\S 7 \mathrm{~b} \quad$ Erhöhte Absetzungen für Einfamilienhäuser, Zweifamilienhäuser und Eigentumswohnungen . . . . . . . . . . . . . . . . . . . .}

$\S 7 \mathrm{E} \quad$ Erhöhte Absetzungen für Baumaßnahmen an Gebäuden zur Schaffung

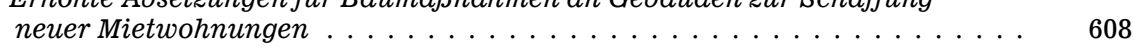

§7d Erhöhte Absetzungen für Wirtschaftsgüter, die dem Umweltschutz dienen . . 608

$\S 7 \mathrm{e} \quad($ weggefallen $) \ldots \ldots \ldots \ldots \ldots$

§7f Bewertungsfreiheit für abnutzbare Wirtschaftsgüter des Anlagevermögens privater Krankenhäuser . . . . . . . . . . . . . . . . . . . . . . . . 608

$\S 7 \mathrm{~g} \quad$ Investitionsabzugsbeträge und Sonderabschreibungen zur Förderung kleiner und mittlerer Betriebe . . . . . . . . . . . . . . . . 608

$\S 7 \mathrm{~h} \quad$ Erhöhte Absetzungen bei Gebäuden in Sanierungsgebieten und städtebau-

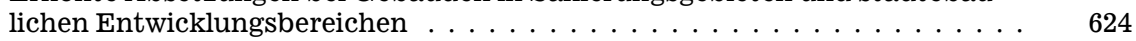

$\S 7 \mathrm{i} \quad$ Erhöhte Absetzungen bei Baudenkmalen . . . . . . . . . . . . . . . 626

$\$ 7 \mathrm{k} \quad$ Erhöhte Absetzungen für Wohnungen mit Sozialbindung . . . . . . . . . 631

\section{4. Überschuss der Einnahmen über die Werbungskosten}

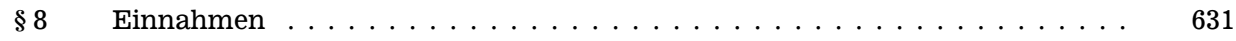

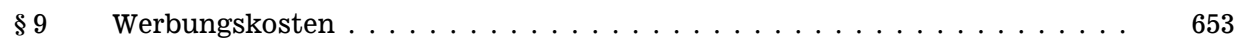

$\S 9 \mathrm{a} \quad$ Pauschbeträge für Werbungskosten $\ldots \ldots \ldots \ldots \ldots$

4a. Umsatzsteuerrechtlicher Vorsteuerabzug

$\S 9 \mathrm{~b} \quad[$ Umsatzsteuerrechtlicher Vorsteuerabzug] $\ldots \ldots \ldots \ldots$

\section{4b. Kinderbetreuungskosten}



\section{Sonderausgaben}









$\S 10 \mathrm{~d}$ Verlustabzug . . . . . . . . . . . . . . . . . . . . 769

$\S 10 \mathrm{e} \quad$ Steuerbegünstigung der zu eigenen Wohnzwecken genutzten Wohnung im eigenen Haus . . . . . . . . . . . . . . . . . . . . . . . . . . . . . .

$\S 10 f \quad$ Steuerbegünstigung für zu eigenen Wohnzwecken genutzte Baudenkmale und Gebäude in Sanierungsgebieten und städtebaulichen Entwicklungs-



$\S 10 \mathrm{~g}$ Steuerbegünstigung für schutzwürdige Kulturgüter, die weder zur Einkunftserzielung noch zu eigenen Wohnzwecken genutzt werden . . . . . . . . . . .

$\S 10 \mathrm{~h} \quad$ Steuerbegünstigung der unentgeltlich zu Wohnzwecken überlassenen Wohnung im eigenen Haus . . . . . . . . . . . . . . . . . . . . . . . . . . . .

$\S 10 \mathrm{i}$ Vorkostenabzug bei einer nach dem Eigenheimzulagengesetz begünstigten



\section{Vereinnahmung und Verausgabung}

$\S 11$ [Zufluss und Abfluss von Einnahmen und Ausgaben] $\ldots \ldots \ldots \ldots \ldots \ldots$

§11a Sonderbehandlung von Erhaltungsaufwand bei Gebäuden in Sanierungsgebieten und städtebaulichen Entwicklungsbereichen $\ldots \ldots \ldots \ldots \ldots$ 
a) Land- und Forstwirtschaft (\$2 Absatz 1 Satz 1 Nummer 1)

$\S 13$ Einkünfte aus Land- und Forstwirtschaft . . . . . . . . . . . . . . . .

$\S 13 \mathrm{a}$ Ermittlung des Gewinns aus Land- und Forstwirtschaft nach Durchschnitts-

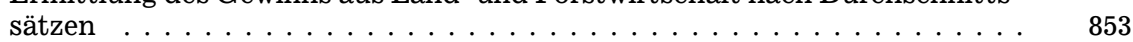

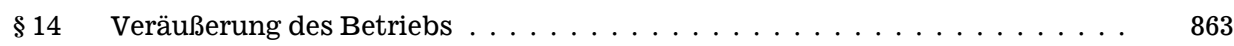

$\S 14 \mathrm{a}$ [Vergünstigungen bei der Veräußerung bestimmter land- und forstwirtschaftlicher Betriebe $] \ldots \ldots \ldots$. . . . . . . . . . . . . 870

b) Gewerbebetrieb ( 2 Absatz 1 Satz 1 Nummer 2 )

$\S 15$ Einkünfte aus Gewerbebetrieb . . . . . . . . . . . . . . . . . . 870

$\S 15$ a Verluste bei beschränkter Haftung . . . . . . . . . . . . . . . . . 1037

$\S 15 \mathrm{~b}$ Verluste im Zusammenhang mit Steuerstundungsmodellen . . . . . . . . . . 1066

$\$ 16 \quad$ Veräußerung des Betriebs . . . . . . . . . . . . . . . . . . . . . 1088

$\S 17$ Veräußerung von Anteilen an Kapitalgesellschaften _ . . . . . . . . . . . 1196

c) Selbständige Arbeit ( 2 Absatz 1 Satz 1 Nummer 3 )

$\S 18 \quad$ [Einkünfte aus selbständiger Arbeit] . . . . . . . . . . . . . . . . . 1256

d) Nichtselbständige Arbeit (§ 2 Absatz 1 Satz 1 Nummer 4)

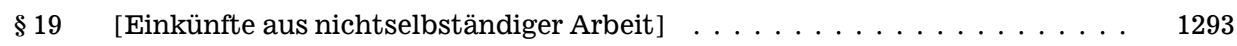

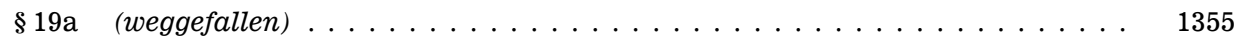

e) Kapitalvermögen ( 2 Absatz 1 Satz 1 Nummer 5)

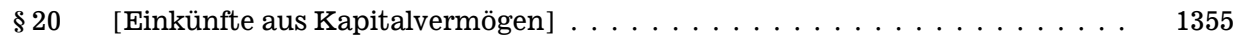

f) Vermietung und Verpachtung ( $\$ 2$ Absatz 1 Satz 1 Nummer 6)

$\S 21$ [Einkünfte aus Vermietung und Verpachtung] . . . . . . . . . . . . . . . . . 1426

g) Sonstige Einkünfte ( 2 Absatz 1 Satz 1 Nummer $\boldsymbol{y}$ )

$\S 22 \quad$ Arten der sonstigen Einkünfte . . . . . . . . . . . . . . . . . . . . 1465

$\S 22 \mathrm{a}$ Rentenbezugsmitteilungen an die zentrale stelle . . . . . . . . . . . . . . . . . . . . . . . . . . . . . .

$\S 23$ Private Veräußerungsgeschäfte . . . . . . . . . . . . . . . . . . 1508

h) Gemeinsame Vorschriften

$\S 24$ [Entschädigungen und nachträgliche Einkünfte] . . . . . . . . . . . . . . . . . . . . . . . . . . . . . . . . . . .

$\S 24 \mathrm{a}$ Altersentlastungsbetrag . . . . . . . . . . . . . . . . . . . . . . . . . . . . . . . . . . . . . . . . . .

$\S 24 \mathrm{~b}$ Entlastungsbetrag für Alleinerziehende . . . . . . . . . . . . . . . . . . . . . 1549

\section{Veranlagung}

$\S 25$ Veranlagungszeitraum, Steuererklärungspflicht . . . . . . . . . . . . 1552

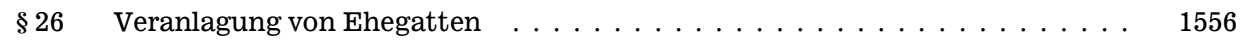

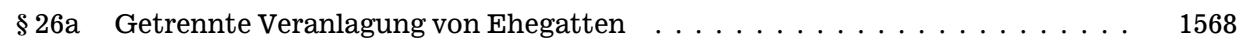

\$26b Zusammenveranlagung von Ehegatten . . . . . . . . . . . . . . . . 1572

§26c Besondere Veranlagung für den Veranlagungszeitraum der Eheschließung $\quad$. 1577

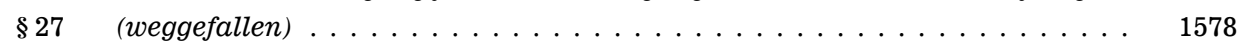

$\S 28$ Besteuerung bei fortgesetzter Gütergemeinschaft . . . . . . . . . . . . . . . . . . . . . . . . . . . . .

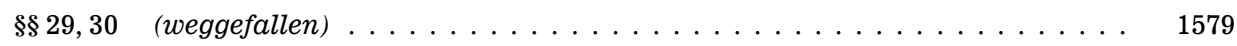

\section{Tarif}

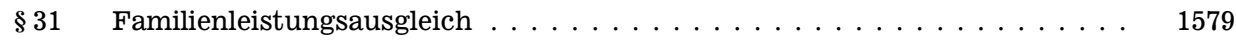

$\S 32 \quad$ Kinder, Freibeträge für Kinder . . . . . . . . . . . . . . . . . . . . . . . . . . . . 1584

$\S 32 \mathrm{a}$ Einkommensteuertarif . . . . . . . . . . . . . . . . . . . . . 1604

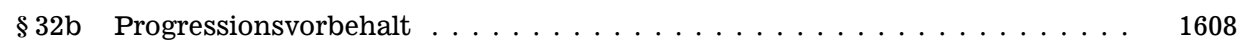


Seite

§32c Tarifbegrenzung bei Gewinneinkünften . . . . . . . . . . . . . . . . . . . 1616

§ 32d Gesonderter Steuertarif für Einkünfte aus Kapitalvermögen . . . . . . . . . . . 1617

$\S 33$ Außergewöhnliche Belastungen . . . . . . . . . . . . . . . . . . 1625

§33a Außergewöhnliche Belastung in besonderen Fällen . . . . . . . . . . . . . 1658

§ 33b Pauschbeträge für behinderte Menschen, Hinterbliebene und Pflegepersonen 1677

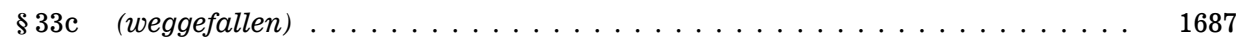

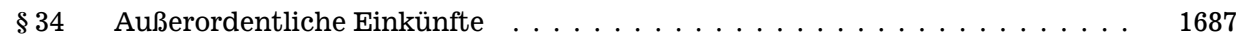

§34a Begünstigung der nicht entnommenen Gewinne . . . . . . . . . . . . . . 1709

§34b Steuersätze bei Einkünften aus außerordentlichen Holznutzungen . . . . . 1757

\section{Steuerermäßigungen}

1. Steuerermäßigung bei ausländischen Einkünften

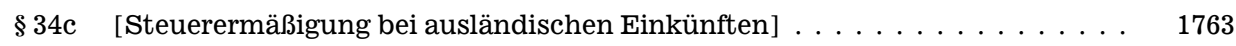

$\S 34 \mathrm{~d}$ Ausländische Einkünfte . . . . . . . . . . . . . . . . . . 1777

2. Steuerermäßigung bei Einkünften aus Land- und Forstwirtschaft

$\S 34 \mathrm{e} \quad$ [Steuerermäßigung bei Einkünften aus Land-und Forstwirtschaft] . . . . . .

2a. Steuerermäßigung für Steuerpflichtige mit Kindern bei Inanspruchnahme erhöhter Absetzungen für Wohngebäude oder der Steuerbegünstigungen für eigengenutztes Wohneigentum

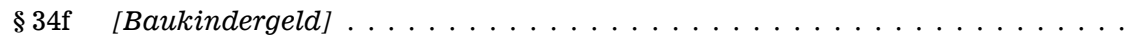

2b. Steuerermäßigung bei Mitgliedsbeiträgen und Spenden an politische Parteien und an unabhängige Wählervereinigungen

§34g [Steuerermäßigung bei Zuwendungen an politische Parteien und unabhängige Wählervereinigungen $] \ldots \ldots \ldots \ldots \ldots \ldots$

3. Steuerermäßigung bei Einkünften aus Gewerbebetrieb

$\S 35$ [Steuerermäßigung bei Einkünften aus Gewerbebetrieb] . . . . . . . . . . .

4. Steuerermäßigung bei Aufwendungen für haushaltsnahe Beschäftigungsverhältnisse und für die Inanspruchnahme haushaltsnaher Dienstleistungen

§35a Steuerermäßigung bei Aufwendungen für haushaltsnahe Beschäftigungsverhältnisse, haushaltsnahe Dienstleistungen und Handwerkerleistungen . .

5. Steuerermäßigung bei Belastung mit Erbschaftsteuer

$\S 35 \mathrm{~b}$ Steuerermäßigung bei Belastung mit Erbschaftsteuer . . . . . . . . . . .

\section{Steuererhebung}

\section{Erhebung der Einkommensteuer}

$\S 36 \quad$ Entstehung und Tilgung der Einkommensteuer . . . . . . . . . . . . . . . . 1811

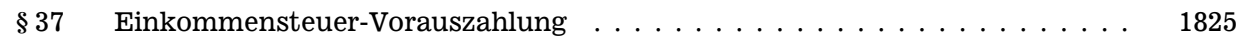

§37a Pauschalierung der Einkommensteuer durch Dritte . . . . . . . . . . . . 1833

§37b Pauschalierung der Einkommensteuer bei Sachzuwendungen . . . . . . . . 1836

2. Steuerabzug von Arbeitslohn (Lohnsteuer)

$\S 38 \quad$ Erhebung der Lohnsteuer . . . . . . . . . . . . . . . . . . . . . . . . . . . . 1842

§ 38a Höhe der Lohnsteuer . . . . . . . . . . . . . . . . . . . . . . . . . . . . . 1851

§ 38b Lohnsteuerklassen, Zahl der Kinderfreibeträge . . . . . . . . . . . . . . . . 1852

$\S 39$ Lohnsteuerabzugsmerkmale . . . . . . . . . . . . . . . . . . 1855

$\S 39$ a Freibetrag und Hinzurechnungsbetrag . . . . . . . . . . . . . . 1860

$\S 39 \mathrm{~b}$ Einbehaltung der Lohnsteuer $\ldots \ldots \ldots \ldots$ 
Seite

§39c Einbehaltung der Lohnsteuer ohne Lohnsteuerabzugsmerkmale . . . . . . . 1871

$\S 39 \mathrm{~d}$ (weggefallen) . . . . . . . . . . . . . . . . . . . . . . . 1874

$\S 39 \mathrm{e} \quad$ Verfahren zur Bildung und Anwendung der elektronischen Lohnsteuer-

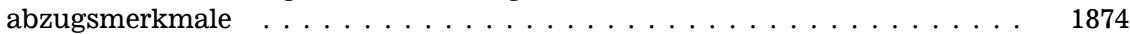

§ 39f Faktorverfahren anstelle Steuerklassenkombination III/V _ . . . . . . . . . . 1880

$\S 40$ Pauschalierung der Lohnsteuer in besonderen Fällen . . . . . . . . . . . . . . 1882

§40a Pauschalierung der Lohnsteuer für Teilzeitbeschäftigte und geringfügig Beschäftigte ........................... 1889

§ 40b Pauschalierung der Lohnsteuer bei bestimmten Zukunftssicherungsleistun-

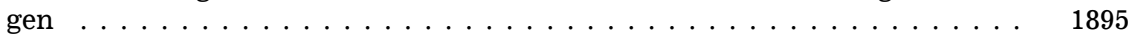

$\S 41 \quad$ Aufzeichnungspflichten beim Lohnsteuerabzug . . . . . . . . . . . . . . . . . 1899

§ 41a Anmeldung und Abführung der Lohnsteuer . . . . . . . . . . . . . . . . . . 1903

$\S 41$ b Abschluss des Lohnsteuerabzugs . . . . . . . . . . . . . . . . . . . . . . 1906

§41c Änderung des Lohnsteuerabzugs . . . . . . . . . . . . . . . . . . . . . . . . . . . . . . . . . . . . . . . . . . . . .

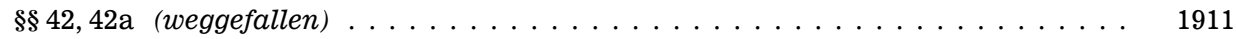

$\S 42 \mathrm{~b}$ Lohnsteuer-Jahresausgleich durch den Arbeitgeber . . . . . . . . . . . . . . 1911

$\S 42 \mathrm{c} \quad$ (weggefallen) . . . . . . . . . . . . . . . . . . . . . . 1914

§ 42d Haftung des Arbeitgebers und Haftung bei Arbeitnehmerüberlassung . . . 1915

$\S 42 \mathrm{e}$ Anrufungsauskunft . . . . . . . . . . . . . . . . . . . . . . . . . . 1933

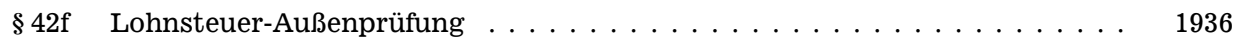

3. Steuerabzug vom Kapitalertrag (Kapitalertragsteuer)

$\S 43 \quad$ Kapitalerträge mit Steuerabzug . . . . . . . . . . . . . . . . . . . . . . 1938

$\S 43 \mathrm{a}$ Bemessung der Kapitalertragsteuer . . . . . . . . . . . . . . . . . . 1950

§ 43b Bemessung der Kapitalertragsteuer bei bestimmten Gesellschaften . . . . . . 1955

$\S 44$ Entrichtung der Kapitalertragsteuer . . . . . . . . . . . . . . . . . . . . . 1960

$\S 44 \mathrm{a}$ Abstandnahme vom Steuerabzug . . . . . . . . . . . . . . . . . 1965

$\S 44 \mathrm{~b}$ Erstattung der Kapitalertragsteuer . . . . . . . . . . . . . . . . . . . 1974

$\S 45$ Ausschluss der Erstattung von Kapitalertragsteuer . . . . . . . . . . . . . . 1977

§ 45a Anmeldung und Bescheinigung der Kapitalertragsteuer . . . . . . . . . . 1978

§ 45b Erstattung von Kapitalertragsteuer auf Grund von Sammelanträgen . . . . 1980

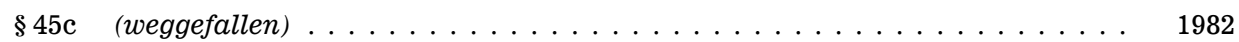

$\S 45 \mathrm{~d}$ Mitteilungen an das Bundeszentralamt für Steuern . . . . . . . . . . . . . . . . . . 1982

$\S 45$ e Ermächtigung für Zinsinformationsverordnung . . . . . . . . . . . . . . . . . 1984

\section{Veranlagung von Steuerpflichtigen mit steuerabzugspflichtigen Einkünften}

$\S 46 \quad$ Veranlagung bei Bezug von Einkünften aus nichtselbständiger Arbeit . . . . . 1985

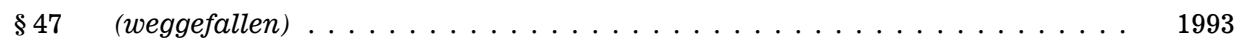

\section{Steuerabzug bei Bauleistungen}

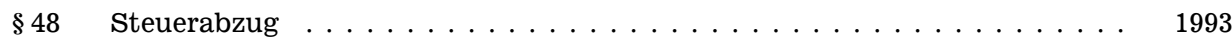

$\S 48 \mathrm{a}$ Verfahren .............................. 2001

$\S 48 \mathrm{~b}$ Freistellungsbescheinigung ........................ 2004

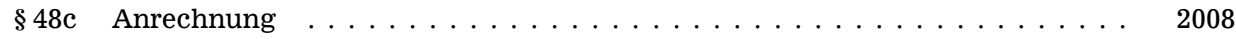

$\S 48 d$ Besonderheiten im Fall von Doppelbesteuerungsabkommen . . . . . . . . 2010

VIII. Besteuerung beschränkt Steuerpflichtiger

$\S 49 \quad$ Beschränkt steuerpflichtige Einkünfte . . . . . . . . . . . . . . . . . . . . 2011

$\S 50$ Sondervorschriften für beschränkt Steuerpflichtige . . . . . . . . . . . 2055

§50a Steuerabzug bei beschränkt Steuerpflichtigen _ . . . . . . . . . . . 2069 
IX. Sonstige Vorschriften, Bußgeld-, Ermächtigungs- und Schlussvorschriften

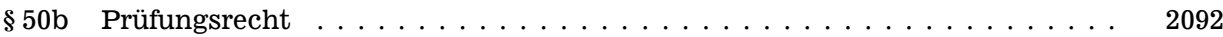

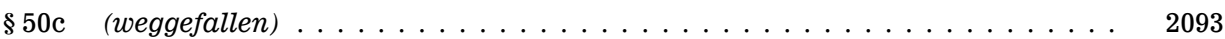

$\S 50 \mathrm{~d}$ Besonderheiten im Fall von Doppelbesteuerungsabkommen und der $\$ \$ 43 \mathrm{~b}$ und $50 \mathrm{~g} \ldots \ldots \ldots \ldots . \ldots \ldots \ldots$

$\S 50 \mathrm{e} \quad$ Bußgeldvorschriften; Nichtverfolgung von Steuerstraftaten bei geringfügiger Beschäftigung in Privathaushalten . . . . . . . . . . . . . . . . 2137

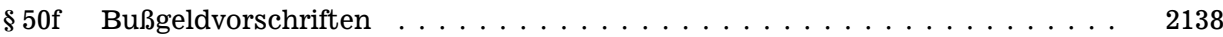

$\S 50 \mathrm{~g}$ Entlastung vom Steuerabzug bei Zahlungen von Zinsen und Lizenzgebühren zwischen verbundenen Unternehmen verschiedener Mitgliedstaaten der Europäischen Union . . . . . . . . . . . . . . . . . . . . .

$\S 50 \mathrm{~h} \quad$ Bestätigung für Zwecke der Entlastung von Quellensteuern in einem anderen Mitgliedstaat der Europäischen Union oder der Schweizerischen Eidgenossenschaft . . . . . . . . . . . . . . . . .

$\S 51$ Ermächtigungen . . . . . . . . . . . . . . . . . . . .

51a Festsetzung und Erhebung von Zuschlagsteuern . . . . . . . . . . . . . . 2178

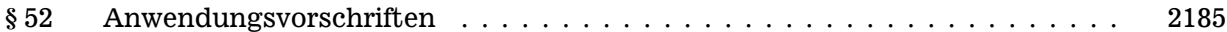

§52a Anwendungsvorschriften zur Einführung einer Abgeltungsteuer auf Kapitalerträge und Veräußerungsgewinne . . . . . . . . . . . . . . . . . .

$\S 52 \mathrm{~b}$ Übergangsregelungen bis zur Anwendung der elektronischen Lohnsteuer-

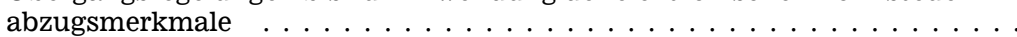

$\S 53 \quad$ Sondervorschrift zur Steuerfreistellung des Existenzminimums eines Kindes in den Veranlagungszeiträumen 1983 bis 1995 . . . . . . . . . . . . . . . . . . .

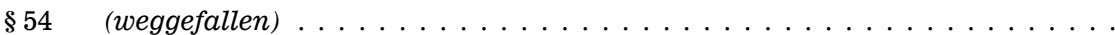

$\S 55$ Schlussvorschriften (Sondervorschriften für die Gewinnermittlung nach § 4 oder nach Durchschnittssätzen bei vor dem 1. Juli 1970 angeschafftem Grund und Boden) . . . . . . . . . . . . . . . . . . . . .

§56 Sondervorschriften für Steuerpflichtige in dem in Artikel 3 des Einigungsvertrages genannten Gebiet . . . . . . . . . . . . . . . . . . . .

$\S 57$ Besondere Anwendungsregeln aus Anlass der Herstellung der Einheit Deutschlands . . . . . . . . . . . . . . . . . . . .

$\S 58$ Weitere Anwendung von Rechtsvorschriften, die vor Herstellung der Einheit Deutschlands in dem in Artikel 3 des Einigungsvertrages genannten Gebiet

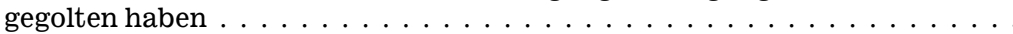

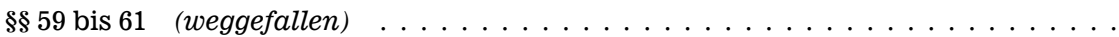

\section{$\mathrm{X}$. Kindergeld}

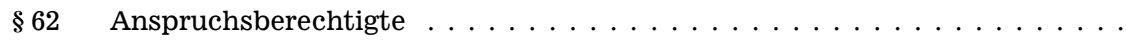

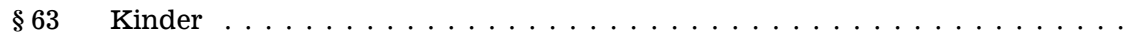

$\S 64$ Zusammentreffen mehrerer Ansprüche . . . . . . . . . . . . . .

$\S 65$ Andere Leistungen für Kinder . . . . . . . . . . . . . . . . . . . . . . . . . . . . 2241

$\S 66$ Höhe des Kindergeldes, Zahlungszeitraum . . . . . . . . . . . . . . . . . . . . 2244

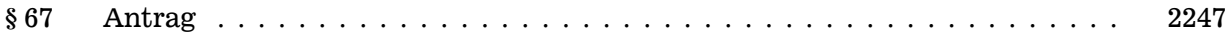

$\S 68$ Besondere Mitwirkungspflichten . . . . . . . . . . . . . . . . . . . 2248

§ 69 Überprüfung des Fortbestehens von Anspruchsvoraussetzungen durch Meldedaten-Übermittlung . . . . . . . . . . . . . . . . 2250

$\S 70 \quad$ Festsetzung und Zahlung des Kindergeldes . . . . . . . . . . . . . . . . . 2250

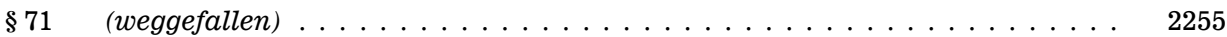

$\S 72$ Festsetzung und Zahlung des Kindergeldes an Angehörige des öffentlichen

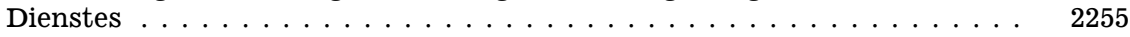

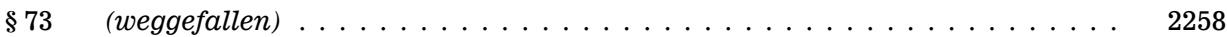

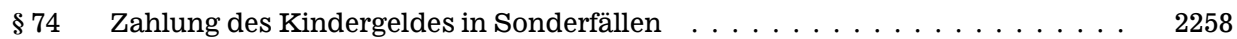


Seite

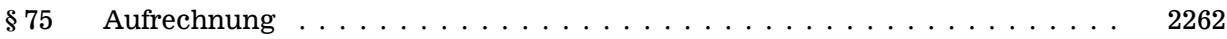

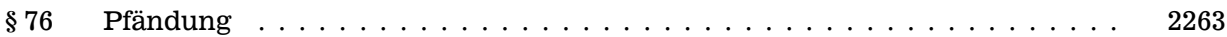

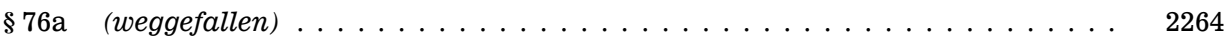

$\S 77$ Erstattung von Kosten im Vorverfahren . . . . . . . . . . . . . . . . . . . . 2264

$\S 78$ Übergangsregelungen . . . . . . . . . . . . . . . . . . . . . . . . . . 2266

\section{Altersvorsorgezulage}

$\$ 79 \quad$ Zulageberechtigte . . . . . . . . . . . . . . . . . . . . . . 2267

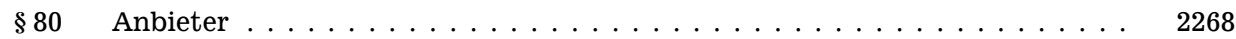

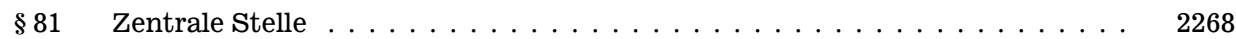

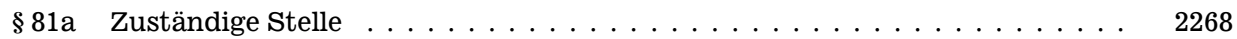

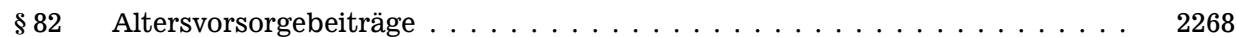

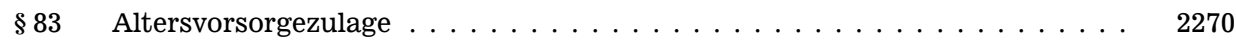

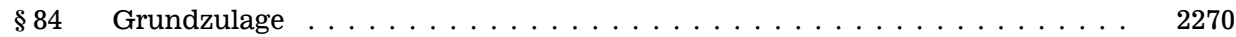

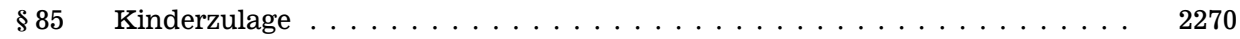

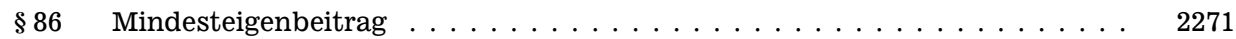

$\S 87$ Zusammentreffen mehrerer Verträge . . . . . . . . . . . . . . . . . . . . . 2274

$\S 88$ Entstehung des Anspruchs auf Zulage . . . . . . . . . . . . . . . . . . . 2274

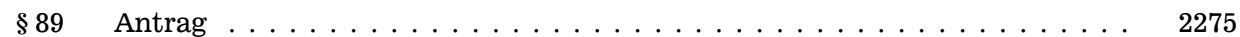

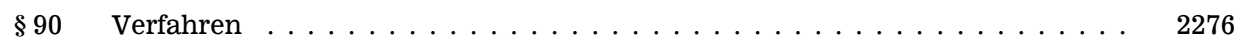

$\S 91 \quad$ Datenerhebung und Datenabgleich . . . . . . . . . . . . . . . . . 2277

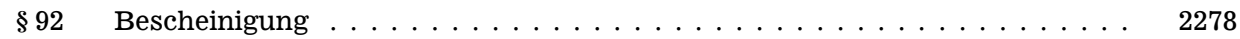

$\S 92$ a Verwendung für eine selbst genutzte Wohnung . . . . . . . . . . . . . 2278

$\S 92 \mathrm{~b}$ Verfahren bei Verwendung für eine selbst genutzte Wohnung . . . . . . . . 2283

$\S 93$ Schädliche Verwendung . . . . . . . . . . . . . . . . . . . . . . . . 2284

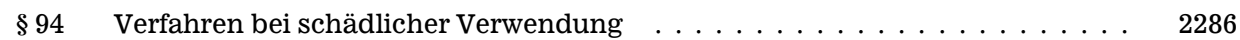

$\S 95$ Sonderfälle der Rückzahlung . . . . . . . . . . . . . . . . . . . . . . . . . 2287

$\S 96$ Anwendung der Abgabenordnung, allgemeine Vorschriften _ . . . . . . . 2288

$\S 97$ Übertragbarkeit . . . . . . . . . . . . . . . . . . . . . . . . . . . . . . . 2289

$\S 98 \quad$ Rechtsweg . . . . . . . . . . . . . . . . . . . . . . . . . . 2289

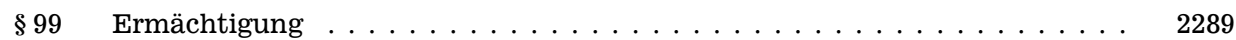

Stichwortverzeichnis . . . . . . . . . . . . . . . . . . . . . 2291 
